\title{
CUANTIFICACIÓN DE RIBOFLAVINA (VITAMINA B2) EN PRODUCTOS LÁCTEOS POR HPLC
}

\section{QUANTIFICATION OF RIBOFLAVIN (VITAMIN B2) IN DAIRY PRODUCTS BY HPLC}

\author{
Carolina Bueno-Solano (1), Olga Nydia Campas-Baypoli (1), Alan Sergio Díaz-García (2), \\ Elda Inés Izaguirre-Flores (2), Wilfrido Verdugo-Zamorano (2), María Isabel Estrada-Alvarado (1), \\ Dalia Isabel Sánchez-Machado (1), Jaime López-Cervantes (1)
}

(1) Programa de Doctorado en Ciencias en Biotecnología, Instituto Tecnológico de Sonora, Sonora, México.

(2) Departamento de Biotecnología y Ciencias Alimentarias, Instituto Tecnológico de Sonora, Sonora, México.

\begin{abstract}
Riboflavin is a water-soluble vitamin of the B complex, and its contribution is essential for the good functioning of the body. Milk is one of the main sources of riboflavin in the human diet; however, in most foods is degraded during conventional thermal processes and storage. In this paper the content of riboflavin in commercial dairy products, milk for direct consumption and yogurt, was determined by high performance liquid chromatographic analysis (HPLC) with fluorescence detection. Sample preparation included an acid hydrolysis (HCL $0.1 \mathrm{~N})$ followed by an enzymatic digestion and a protein precipitation at the end (TCA 100\%). For the HPLC analysis an isocratic bomb program was used (0.005 $\mathrm{M}$ ammonium acetate-methanol, 72:28 v/v). Riboflavin concentrations in yogurt samples were $0.289-3.078 \mu \mathrm{gB} 2 / \mathrm{g} \mathrm{DW}$, while in the ultra-high-temperature pasteurized milk and pasteurized milk was 0.61-13.64 $\mu \mathrm{gB} 2 / \mathrm{g} \mathrm{DW}$ and 11.73-15.41 $\mu \mathrm{gB} 2 / \mathrm{g} \mathrm{DW}$, respectively. Milk for direct consumption and yogurt are good sources of riboflavin; its regular consumption helps to satisfy daily requirements.
\end{abstract}

Key words: Riboflavin; HPLC; yogurt; milk.

Este trabajo fue recibido el 2 de Noviembre de 2008 y aceptado para ser publicado el 24 de Marzo de 2009.

\section{INTRODUCCIÓN}

Las vitaminas son un grupo de compuestos orgánicos que se encuentran en pequeñas cantidades en muchos alimentos, las que son esenciales para lograr un crecimiento y funcionamiento óptimo del cuerpo humano (1). Las funciones que desempeñan las vitaminas son diversas, actúan como coenzimas, antioxidantes, en la regulación genética y en funciones especializadas (2). Las vitaminas se dividen en dos grupos, hidrosolubles y liposolubles. Dentro de las hidrosolubles se encuentran las vitaminas del complejo B formado por la tiamina, riboflavina, niacina, piridoxina, ácido pantoténico, biotina, cobalamina y ácido fólico (3).

La sustancia matriz de los compuestos de la familia de la riboflavina es la 7,8-dimetil-10(1'-ribitil) isoaloxacina y a todos los derivados se les aplica el nombre de flavinas (figura 1) (2). Generalmente, se encuentra fosforilada integrando las coenzimas flavín mononucleótido y flavín adenín dinucleótido; las cuales funcionan como coenzimas de numerosas enzimas flavina-dependientes que catalizan diversos procesos de oxidación-reducción (4). Ambas se convierten fácilmente en riboflavina por la acción de las fosfatasas presentes en los alimentos y en el aparato digestivo. Esta vitamina es sintetizada solamente por microorganismos y vegetales, los animales no pueden sintetizarla. La riboflavina es esencial para la prevención de queilosis, dermatitis seborreica, vascularización cornea, entre otras.

Debido a las altas concentraciones de vitaminas del complejo B presentes en carnes y leche, estos alimentos son considerados como las mejores fuentes naturales de riboflavina en la dieta del hombre en todo el mundo (5). Existe una amplia gama de productos alimenticios obtenidos a partir de la leche, algunos de ellos implican el uso de la leche entera y otros sólo porciones de ésta separadas mediante distintas operaciones. Existen mu- 
chas razas de bovino que se destinan a la producción de leche en todo el mundo, sin embargo, en México se utilizan más comúnmente la raza Holstein Freisian y Jersey. Las leches disponibles en el mercado para consumo directo pueden ser de vida útil corta (leche pasteurizada, estable de 3-5 días bajo refrigeración) y larga (leche ultrapasteurizada, estable durante meses a temperatura ambiente) (6). El yogurt es un producto lácteo fermentado que resulta del crecimiento de las bacterias lácticas Streptococcus termophilus y Lactobacilus bulgarias en la leche, debe resultar un líquido suave y viscoso, o un gel suave y delicado, con sabor característico (7). Una dieta rica en leche y productos lácteos es una excelente fuente de proteínas, calcio, magnesio, fósforo y riboflavina $(8,9)$. Sin embargo, una pérdida considerable de riboflavina ocurre cuando el alimento es expuesto a la luz. La fotodegradación de la riboflavina en productos lácteos depende de diversos factores, tales como, intensidad de la luz, tiempo de exposición y tipo de contenedor (10).

Entre los métodos utilizados para la identificación y cuantificación de riboflavina en los alimentos se encuentran los fluorimétricos, microbiológicos y espectrofotométricos. Sin embargo, algunos de ellos tienen baja especificidad y un tiempo de análisis largo (11). La cromatografía líquida de alta resolución (HPLC) es una técnica ampliamente utilizada en la industria farmacéutica para un análisis rápido de vitaminas, debido a su alta sensibilidad y especificidad. En comparación con la detección ultravioleta, la de fluorescencia es más rápida, selectiva y detecta cantidades más pequeñas del analito (12-15). El objetivo de este trabajo fue la cuantificación de riboflavina en productos lácteos comerciales tales como leches para consumo directo y yogurt por HPLC con detección por fluorescencia. Previamente, la vitamina fue extraída con ácido diluido en caliente, seguida de una extracción enzimática y precipitación de las proteínas.

\section{MATERIAL Y MÉTODOS Reactivos y materiales}

El estándar de riboflavina fue obtenido de Sigma (St. Louis, MO, USA); metanol grado HPLC de EDM (Darmstadt, Germany), acetato de amonio de Merck (Darmstadt, Germany). Ácido acético, ácido clorhídrico, etanol y acetato de sodio de Fermont (Monterrey, Nuevo León, México). Ácido tricloroacético de Fluka, (Seelze, Germany). Enzima claradiastasa de Fluka (Buchs, Switzerland). Todas las soluciones fueron preparadas con agua ultrapura purificada en un sistema NANOpure Diamond UV de Barnstead International (Dubuque, Iowa, USA).

Las soluciones preparadas fueron: ácido clorhídrico $0,1 \mathrm{~N}$; acetato de amonio $0,005 \mathrm{M}$; acetato de sodio $2,5 \mathrm{M}$; enzima claradiastasa $6 \%$ en agua. La solución del estándar de riboflavina $(30 \mu \mathrm{g} / \mathrm{ml})$ se preparó disolviendo $1,5 \mathrm{mg}$ de riboflavina en $50 \mathrm{ml}$ de ácido acético $0,02 \mathrm{~N}$. El estándar se protege de la luz y se almacena

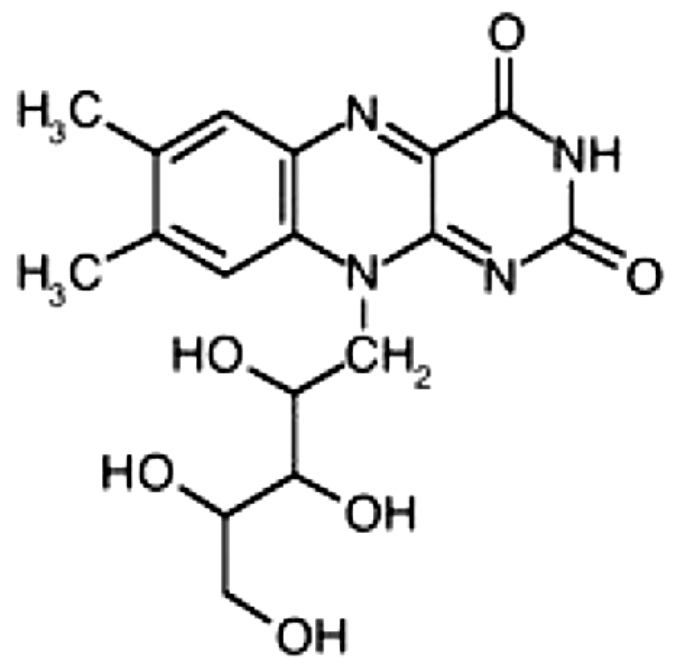


en refrigeración. La solución de claradiastasa se prepara el mismo día de su uso.

\section{Muestras}

Se analizaron 8 muestras de yogurt natural, 4 de leche pasteurizadas y 8 de leche ultrapasteurizadas. Las muestras provenían de distintas empresas procesadoras del país y fueron adquiridas en supermercados de la localidad (Cajeme, Sonora, México). Las descripciones de cada muestra se mencionan en las tablas 1 y 2, para el yogurt y las leches, respectivamente. Así mismo, las muestras se mantuvieron en refrigeración y protegidas de la luz hasta su uso.

\section{Extracción de riboflavina}

Se realizó una hidrólisis ácida y una hidrólisis enzimática, siguiendo el método propuesto por SánchezMachado et al., (2004) (13) con algunas modificaciones. Específicamente, $5 \mathrm{~g}$ de cada muestra se colocan en un frasco color ámbar, $10 \mathrm{ml}$ de $\mathrm{HCl} 0,1 \mathrm{~N}$ fueron adicionados, se mezclan y se hidroliza a $100{ }^{\circ} \mathrm{C}$ por $30 \mathrm{~min}$. Se enfría a temperatura ambiente y el $\mathrm{pH}$ se ajusta a 4,3-4,7

\section{TABLA 1}

\section{Descripción de las muestras de yogurt natural seleccionadas}

\begin{tabular}{cl} 
Muestra & \multicolumn{1}{c}{ Descripción } \\
\hline 1 & Yogurt natural líquido para beber \\
2 & Yogurt natural fortificado con vitaminas y minerales \\
3 & Yogurt natural cremoso \\
4 & Yogurt sin grasa natural con antioxidantes \\
5 & Yogurt natural cremoso light \\
6 & Yogurt natural sin grasa endulzado con edulcorantes. \\
7 & Bebida láctea fermentada sabor natural con Lactobacilos vitakasei \\
8 & Yogurt natural sin grasa fortificado con vitaminas
\end{tabular}

TABLA 2

Descripción de las muestras de leche ultrapasteurizada y pasteurizada seleccionadas

Muestra

Ultrapasteurizada

1

2

3

4

5

6

7

8

Pasteurizada

1

2

3

4
Descripción

Fortificada con extracalcio
Semidescremada
Light
Entera
Deslactosada
Entera
Light

Entera con omega 3

Light

Entera

Deslactosada

Semidescremada 
con acetato de sodio $2,5 \mathrm{M}$. Posteriormente, se le adiciona $1,25 \mathrm{ml}$ de enzima claradiastasa $6 \%$, se incuba en un horno eléctrico a $50{ }^{\circ} \mathrm{C}$ por 3 horas. Después se adiciona $1 \mathrm{~g}$ de ácido tricloroacético al $100 \%$ y se sonifica por 20 min, se filtra al vacío a través de papel Whatman No, 41 (Maidstone, England). El filtrado se diluye a $25 \mathrm{ml}$ con agua ultrapura. La solución resultante fue filtrada con una membrana de poro $0,45 \mu \mathrm{m}$ y se inyecta en el equipo HPLC. El contenido de riboflavina en las muestras fue expresado en base seca.

\section{Equipo y condiciones cromatográficas}

El sistema HPLC (VARIAN, Melbourne, Victoria, Australia) está equipado con; autoinjector 410, desgasificador (Metachem), bomba 230, detector de fluorescencia 363 y un sistema controlador de datos Galaxie Workstation. El análisis cromatográfico se realizó utilizando una columna analítica (150 mm x 4,6 mm, $5 \mu \mathrm{m}$ ) Chromsep SS C18 con un guarda columnas SS 10 x 3 mm (Varian, Melbourne, Victoria, Australia). Las condiciones del análisis cromatográfico fueron las siguientes: la fase móvil fue una mezcla de acetato de amonio 0,005M-metanol $(72: 28 \mathrm{v} / \mathrm{v})$; velocidad de flujo $1,0 \mathrm{ml} / \mathrm{min}$; temperatura de columna de $28^{\circ} \mathrm{C}$. La detección por fluorescencia se realizó a $\lambda$ ex $=450 \mathrm{~nm}$ y $\lambda$ em $=525 \mathrm{~nm}$, y el tiempo total de análisis fue de 12 min.
La identificación de la riboflavina en las muestras se llevó a cabo comparando los tiempos de retención del pico del estándar y con el de la muestra. Para la determinación del tiempo de retención del estándar se inyectó una concentración conocida por triplicado. Otro factor que permite confirmar la presencia de la riboflavina es la longitud de onda utilizada, tanto de excitación como de emisión, ya que son específicas para la riboflavina. La identificación con un detector de fluorescencia es más sensible y selectiva que con un detector UV-Vis, la cual puede presentar interferencias.

\section{RESULTADOS Y DISCUSIÓN}

Para cuantificar el contenido de riboflavina en las muestras se generó una curva de calibración en base a una concentración conocida de riboflavina $(30 \mu \mathrm{g} / \mathrm{ml})$, a partir de esta se prepararon ocho concentraciones conocidas, seleccionadas en base al contenido de riboflavina presente en las muestras. La curva es lineal en el rango de 0,012 y $0,6 \mu \mathrm{g} \mathrm{B}_{2} / \mathrm{ml}$, y suministrando una ecuación de la recta $(y=2497 x+3,879)$ con un coeficiente de correlación apropiado $\left(\mathrm{r}^{2}=1\right)$. La variable independiente (x) es la concentración y la variable dependiente (y) es el área del pico del cromatograma. En la figura 2 (A, B y C) se muestran los cromatogramas del estándar, yogurt y leche ultrapasteurizada, respectivamente.

\section{FIGURA 2}

Cromatograma de la riboflavina en, (A) estándar, (B) yogurt y (C) leche ultrapasteurizada
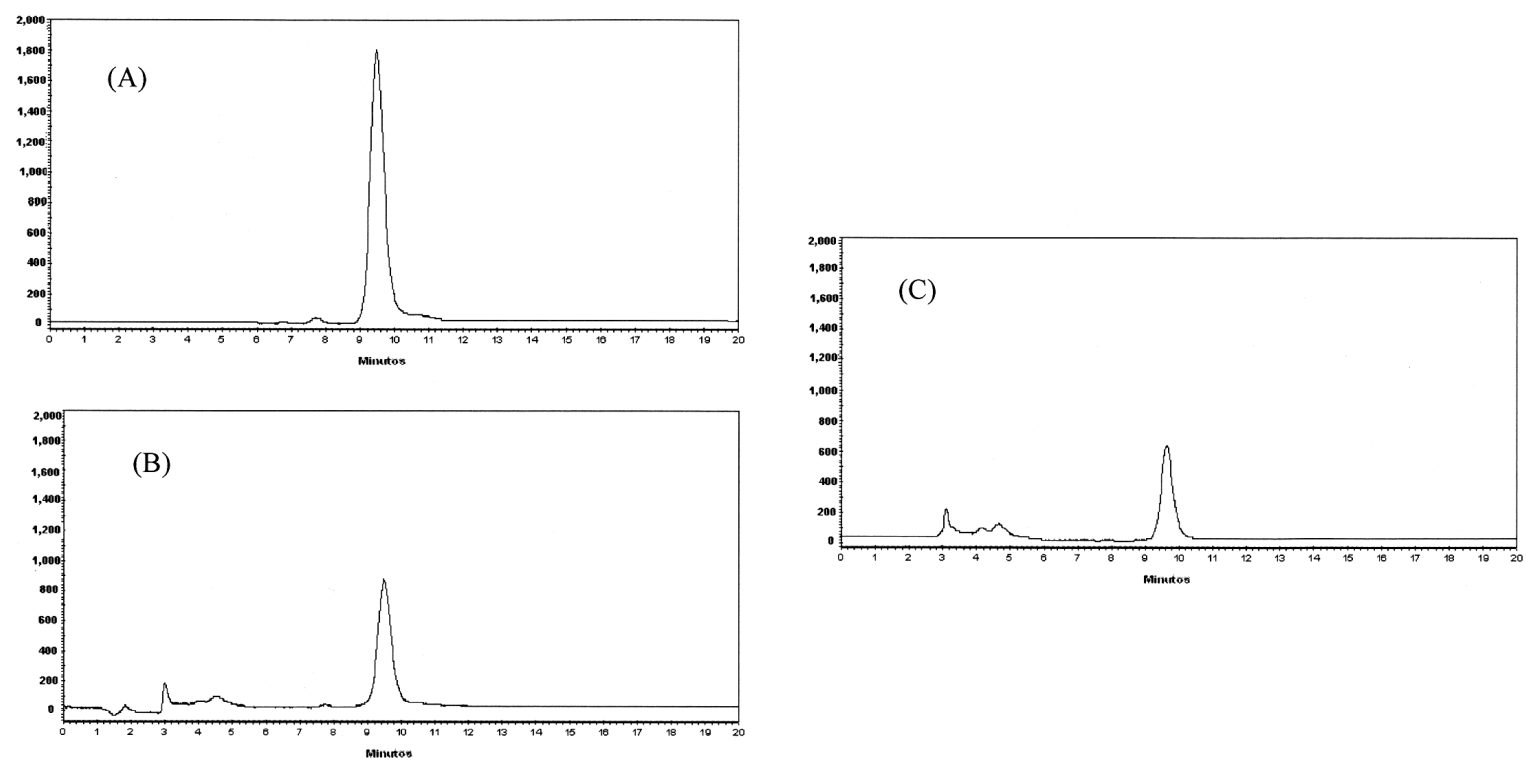
La humedad es un importante indicador de eficiencia y calidad de producto, debido a que entre menos humedad tenga el producto su vida de anaquel será más larga (16). El contenido de humedad en el yogurt natural se encuentra en un rango de 75,69 - 88,41 (g/100 g BH). Estos valores del contenido de humedad son similares (85.7 g/100 g BS) a los reportados en yogurt natural (3). El contenido de humedad en las muestras de leche ultrapasteurizada esta en un rango de 87,29 a 90,54 (g/100 g BH), mientras que en la pasteurizada es de 88,99 a 91,02 (g/100 g BH).
En la tabla 3, se presentan los valores encontrados de riboflavina en las diferentes muestras de yogurt comercial. El contenido de riboflavina en el yogurt natural comercial se encuentra en el rango de 0,289 a 3,078 $\mu \mathrm{g} / \mathrm{g}$ BS. Diversos autores han reportado contenidos similares de riboflavina en yogurt natural $(2,05 \mu \mathrm{g} / \mathrm{g} \mathrm{BS})(17)$, yogurt aromatizado (3 $\mu \mathrm{g} / \mathrm{g}$ BS) (18), así como yogurt con frutas (2 $\mu \mathrm{g} / \mathrm{g} \mathrm{BS})$ (19). Mientras que nuestros resultados difieren de los logrados por otros autores para el yogurt natural elaborado con leche semidescremada $(14.3 \mu \mathrm{g} / \mathrm{g}$ BS) y el yogurt elaborado con leche descremada (15.5

\section{TABLA 3}

\section{Concentración de riboflavina en de yogurt comercial}

\begin{tabular}{cc} 
Muestra & Concentración $(\boldsymbol{\mu g} / \mathbf{g} \mathbf{B S})$ \\
1 & 0,29 \\
3 & 0,52 \\
4 & 0,38 \\
5 & 2,75 \\
6 & 1,54 \\
7 & 3,08 \\
8 & 1,39 \\
\hline
\end{tabular}

\section{TABLA 4}

Concentración de riboflavina en leche ultrapasteurizada y pasteurizada

Muestra

\section{Leche ultrapasteurizada}

1

2

3

4

5

6

7

8

Leche pasteurizada
Concentración $(\mu \mathrm{g} / \mathrm{g} \mathrm{BS})$
11,64

13,64

0,61

11,68

12,07

13,52

11,94

15,41

11,72

14,57

13,14 
$\mu \mathrm{g} / \mathrm{g}$ BS) (20).

En la tabla 4, se muestra el contenido de riboflavina en las muestras de leche ultrapasteurizada y pasteurizada. En los análisis realizados a las muestras de leche ultrapasteurizada la mayor concentración de riboflavina fue de $13,64 \mu \mathrm{g} / \mathrm{g}$ BS y la menor de $0,61 \mu \mathrm{g} / \mathrm{g}$ BS. En las muestras de leche pasteurizada el contenido de riboflavina encontrada esta en un rango de 11,73 a $15,41 \mu \mathrm{g} / \mathrm{g}$ BS. En otras investigaciones donde se identificaron y cuantificaron a las vitaminas del complejo B en leche, encontrando un contenido de riboflavina de $1,42 \mu \mathrm{g} / \mathrm{g}$ BS, valor que está por debajo de la mayoría de las muestras analizadas en esta investigación (21). Por otra parte, se ha reportado el contenido de riboflavina en leche entera $(1,61 \mu \mathrm{g} / \mathrm{g}$ BS$)$ y desnatada $(1,71 \mu \mathrm{g} / \mathrm{g} \mathrm{BS})$, los cuales están notablemente por debajo de los obtenidos en esta investigación. Igualmente, se determinó por HPLC con detección ultravioleta visible la concentración de riboflavina en leche, encontrando de 1,16 a 1,31 $\mathrm{gg} / \mathrm{g}$ BS (22). Otros autores, han cuantificado vitaminas hidrosolubles en leche en formulas infantiles utilizando biosensores (23), reportando $11,8 \mu \mathrm{g} / \mathrm{g}$ BS de riboflavina, valor que es similar a los obtenidos en esta investigación. La diferencia que se presenta en las concentración de riboflavina en la muestras puede deberse al tipo leche analizada, ya que algunas de ellas están adicionadas con vitaminas, otras son light, deslactosadas, semidescremadas o enteras. También el contenido de riboflavina se ve influenciado por las razas de vacas utilizadas para la obtención de la leche. La riboflavina es estable a los procesos térmicos, tal es el caso, de la pasteurización, evaporación o condensación, sin embargo, pueden ocurrir ligeras variaciones en el contenido de esta vitamina (5). La exposición a la luz, especialmente a longitudes de onda por debajo de los $500 \mathrm{~nm}$, puede destruir la riboflavina, así como también la presencia de oxígeno $(24,25)$. El material de empaque influye en el contenido de riboflavina, ya que éste protege a la leche de la luz. Ambos, la luz y el oxígeno, pueden provocar la decoloración, formación o pérdida de sabor, así como también la pérdida de nutriente. Considerando que la leche de vaca recién ordeñada contiene $16.35 \mu \mathrm{g}$ $\mathrm{B}_{2} / \mathrm{g}$ BS y la leche pasteurizada $13.71 \mu \mathrm{g} \mathrm{B}_{2} / \mathrm{g}$ BS se tendría una pérdida de riboflavina durante su procesamiento del $16 \%$, por su parte la leche ultrapasteurizada con un contenido de $12.41 \mu \mathrm{g} \mathrm{B}_{2} / \mathrm{g}$ BS tendría una degradación del $24 \%$. Así mismo, los valores reportados en este trabajo para yogurt y leches procesadas son comparables con aquellos reportados en otros productos que componen la dieta tradicional, tal es el caso de sardinas enlatadas (6.25 $\mu \mathrm{g} / \mathrm{g} \mathrm{BS})$, pan blanco (5.07 $\mu \mathrm{g} / \mathrm{g}$ BS), huevo (12 $\mu \mathrm{g} / \mathrm{g} \mathrm{BS})$, pero difieren para la carne de vaca (48.12 $\mu \mathrm{g} / \mathrm{g}$ BS) (20).
Los requerimientos de riboflavina establecidos son 0,4 mg/día para niños de 7 a 12 meses de edad y de 1,3 $\mathrm{mg}$ /día para adultos, en base a los resultados obtenidos en esta investigación un niño de 7 a 12 meses tendría que ingerir aproximadamente $300 \mathrm{ml}$ de leche pasteurizada al día para cumplir con los requerimientos (26).

\section{CONCLUSIONES}

Las leches para consumo directo (pasteurizada o ultrapasteurizada) y el yogurt natural son alimentos ricos en riboflavina por lo que su consumo moderado permite satisfacer los requerimientos diarios tanto para niños como para adultos. El método HPLC utilizado permite el análisis rápido y preciso de la riboflavina.

\section{RESUMEN}

La riboflavina $\left(\mathrm{B}_{2}\right)$ es una vitamina hidrosoluble del complejo B, cuyo aporte nutricional es imprescindible para el buen funcionamiento del organismo. La leche es una de las principales fuentes de riboflavina en la dieta humana, sin embargo, en la mayoría de los alimentos se degrada durante los procesos térmicos convencionales $y$ en el almacenamiento. En el presente trabajo se determinó el contenido de riboflavina en productos lácteos comerciales, leches para consumo directo y yogurt, por cromatografía líquida de alta resolución (HPLC) con detección por fluorescencia. La preparación de la muestra incluye una hidrólisis ácida $(\mathrm{HCl} 0.1 \mathrm{~N})$, seguida por una digestión enzimática, y por último, la precipitación de proteínas (TCA $100 \%$ ). En el análisis cromatográfico se utilizó un sistema isocrático (acetato de amonio $5 \mathrm{mM}$ metanol, $72: 28 \mathrm{v} / \mathrm{v}$ ). El contenido de riboflavina de las muestras de yogurt estuvo en el rango de 0,289 - 3,078 $\mu \mathrm{g} / \mathrm{g} \mathrm{BS}$, mientras que en la leche ultrapasteurizada y pasteurizada de $0,61-13,64 \mu \mathrm{g} / \mathrm{g}$ BS y 11,73-15,41 $\mu \mathrm{g} /$ $\mathrm{g}$ BS, respectivamente. Las leches para consumo directo y el yogurt comercial son fuentes de riboflavina, y su consumo regular puede ayudar a satisfacer los requerimientos diarios.

Palabras clave: Riboflavina; HPLC; yogurt; leche.

Dirigir la correspondencia a:

Profesor

Jaime López-Cervantes

Programa de Doctorado

en Ciencias en Biotecnología.

Instituto Tecnológico de Sonora.

5 de Febrero 818 Sur, CP 85000, Cd. Obregón

Sonora, México.

Teléfono: +52-6444100900

Fax: $+52-6444109001$

E-mail: jlopezc@itson.mx 


\section{BIBLIOGRAFÍA}

1. Ekinci R, Kadakal C. Determination of seven water soluble vitamins in tarhana, a traditional turkish cereal food, by high performance liquid chromatography. Acta Chromatogr 2005; 15: 289-297.

2. Gregory III, JF. Vitaminas. En: Química de los alimentos. Acribia (Ed.). España 2000; pp. 634643, 685-689.

3. Fox BA, Cameron AG. Ciencia de los alimentos, nutrición y salud. Limusa. México 2002, pp. 94, 271-285.

4. Valls F, Sancho MT, Fernández-Muiño MA, Checa MA. Determination of total riboflavin in cooked sausage. J Agric Food Chem 1999; 47: $1067-$ 1070.

5. Severo Silva L, Trevisan MG, Rath S, Poppi RJ, Reyes FGR. Chromatographic determination of riboflavin in the presence of tetracyclines in skimmed and full cream milk using fluorescence detection. J Braz Chem Soc 2005; 16: 1174-1178.

6. Ordóñez JA, Cambero MI, Fernández L, García ML, García de Fernando G, De la Hoz L, Selgas MD. Tecnología de los alimentos. Vol. II. Alimentos de origen animal. Síntesis. Madrid 1998; pp. 64-67.

7. García Garibay M, Revah S, Gómez L. Productos lácteos, En: Biotecnología Alimentaria. Limusa. México 2002; pp. 153-159, 163-174.

8. Cornejo EV, Fernández GE, Castro Ch G, Vargas FS, Henríquez RC. Estudio sobre dilución de tres tipos leche en polvo en familias pertenecientes a niveles socioeconómico: abc1, c2 y c3 de Santiago. Rev Chil Nutr 2007; 34 (4):353-363.

9. Krešić G, Šimundića B, Mandićb ML, KendQelc G, Žeželjc SP. Daily menus can result in suboptimal nutrient intakes, especially calcium, of adolescents living in dormitories. Nutr Res 2008; 28: 156-165.

10. Lee KH, Yung MY, Kim SY. Effects of ascorbic acid on the light-induced riboflavin degradation and color changes in milk. Department of food Science and Technology. J Agric Food Chem 1998, 46: 407-410.

11. Esteve MJ, Farré R, Frívola A, García-Cantabella JM. Simultaneous determination if thiamine and riboflavin in mushrooms bye Liquid Chromatography. J Agric Food Chem 2001; 49: 1450-1455.

12. Dawson KR, Unklesbay NF, Hedrick HB. HPLC determination of riboflavin, niacin, and thiamin in beef, pork and lamb after alternate heat-processing methods. J Agric Food Chem 1998;36:1176-1179.

13. Sánchez-Machado DI, López-Cervantes J, López-
Hernández J, Paisero-Losada P. Simultaneous determination of thiamine and riboflavin in edible marine seaweeds by high performance liquid chromatography. J Chromatogr Sci 2004; 42: 1-4.

14. Ang CYW, Moseley FA. Determination of thiamin and riboflavin in meat and meat products by high pressure liquid chromatography. J Agric Food Chem 1998; 28 (3): 483-486.

15. Gliszczynska-Swiglo A, Koziolowa A. Chromatographic determination of riboflavin and its derivatives in food. J Chromatogr A 2000; 881: 285-297.

16. Badui S. Química de los alimentos. Pearson. México 2006; pp. 363-366, 379-381, 628.

17. Martínez JA. Fundamentos teórico-prácticos de nutrición y dietética. McGraw-Hill Interamericana de España. Madrid. 1998; pp. 382-388.

18. Bender DA. Introducción a la nutrición y el metabolismo. Acribia,S.A. España 1995; pp.316.

19. Matix-Verdú J, Carazo-Marín E. Nutrición para educadores. Diaz de Santos, S.A. España 1995; pp.415.

20. Mahan LK, Escott-Stump S. Nutrición y dietoterapia de, Krause. McGraw-Hill Interamericana. México 2000; pp.1050-1051.

21. Albalá-Hurtado S, Veciana-Nogués MT, IzquierdoPulido M, Mariné-Font A. Determination of water soluble vitamins in infant milk by high-performance liquid chromatography. J Chromatogr A 1997; 778: 247-253.

22. Muñoz A, Ortiz R, Murcia MA. Determination by HPLC of changes in riboflavin levels in milk on nondairy imitation milk during refrigerated storage. Food Chem 1994; 49: 203-206.

23. Gao Y, Guo S, Gokavi S, Chow A, Sheng Q, Guo M. Quantification of water-soluble vitamins in milk-based infant formulae using biosensor-based assays. Food Chem 2008; 110: 769-776.

24. Zygoura P, Moyssiadi T, Badeka A, Kondyli E, Savvaidis I, Kontominas MG. Shelf life of whole pasteurized milk in Greece: effect of packaging material. Food Chem 2004; 87: 1-9.

25. Becker EM, Christensen J, Frederiksen CS, Haugaard VK. Front-face fluorescence spectroscopy and chemometrics in analysis of yogurt: rapid analysis of riboflavin. J Dairy Sci 2003; 86: 25082515.

26. FAO/WHO. Joint FAO/WHO Expert Consultation on human vitamin and mineral requirements. FAO, Bangkok, Thailand, September, 1998. 